\title{
Design and development of self-diagnosis composites containing electrically conductive phase
}

\author{
Hideaki MATSUBARA, Yoshiki OKUHARA and Hiroaki YANAGIDA \\ Japan Fine Ceramics Center, 2-4-1 Mutsuno, Atsuta-ku Nagoya, 456-8587 Japan TEL: x81-052-871- \\ 3500 Fax: x81-052-871-3599, e-mail: matsubara@jfcc.or.jp \\ (Received 16, August 2000 Accepted 30, November 2000)
}

\begin{abstract}
The electrically conductive fiber reinforced plastics (FRP) and ceramics matrix composites (CMC) have been designed and fabricated in order to introduce the self-diagnosis function in these materials. The electrical conductivity was achieved by adding conductive particles into the matrices of these composites. The FRP with percolation structure consisting of carbon particles had liner response of resistance to the tensile strain and high sensitivity in the wide strain range. The FRP embedded in mortar block successfully diagnosed micro-crack formation and propagation, showing that the self-diagnosis function of the FRP has the ability to monitor the health condition of concrete structures. The CMC materials containing TiN particles as a conductive phase indicated not only fine response of resistance to the applied strain but also resistance increase with increasing number of repetitions during cyclic bending test. These results suggest that these composites can be brought to many industrial applications concerning the self-diagnosis of deformation and damage in structural materials.
\end{abstract}

Key words: Self-diagnosis, Composite, Resistance, FRP, CMC

\section{INTRODUCTION}

The overall deterioration of structural materials due to aging or disaster has resulted in the expansion of demand for non-destructive evaluation of health condition. Recently, the health monitoring technique utilizing the structural materials with function to diagnose own conditions, ${ }^{1}$ so-called self-diagnosis materials or intelligent materials, has been attracting attentions. Some methods of the fracture detection in the fiber reinforced plastics (FRP) which were applied to structural materials as a reinforcement have been proposed..$^{2-8}$ Muto et al. have reported that the resistance change in carbon-fiber glass-fiber reinforced plastics (CFGFRP) were applied to monitor the fatal fracture. ${ }^{2}$ Although the CFGFRP showed appreciable resistance change in the strain range above $0.7-1.5 \%$ due to fracture in carbon fiber, the detectable strain is relatively large considering the diagnosis of local damage in structural materials such as concrete. An acoustic emission method and fiber optics sensor have been researched with the aim of applying to health monitoring, but these complicated systems are expensive. It is therefore necessary to develop the self-diagnosis materials showing higher sensitivity in the small strain region and to achieve simple health monitoring system.

In the present work, the electrically conductive composites having continuous structure of conductive particles, so-called percolation structure, were designed and fabricated in the FRP and the ceramics matrix composites (CMC). These selfdiagnosis functions were evaluated from the measurement of resistance changes with applied strain in normal or cyclic loading tests. The practicability of the function was examined in bending tests for mortar specimen embedding FRP. 


\section{SELF-DIAGNOSIS FUNCTION OF FRP}

Figure 1 is the schematic drawing of the structural design for conductive FRP which basically consists of vinyl ester resin (Showa High Polymer Co., Ltd. RIPOXY R-804) and glass fiber (Asahi glass fiber Co, Ltd. ER2220). The carbon fiber (pitch-based CF, Toho Rayon Co., Ltd. BESFIGHT UM63) introduced in replacement of a part of glass fiber as shown in Fig. 1 (a) forms conductive path and enhances strength of the composite in the longitudinal direction. The carbon particles (graphite, SEC Co., Ltd. SPG5) dispersed in a part of matrix create conductivity due to the formation of percolation structure as shown in Fig.1 (b). The composite containing carbon particles was indicated by carbon-particles glass-fiber reinforced plastics (CPGFRP).

The self-diagnosis functions of these materials were evaluated by simultaneous measurements of stress and electrical resistance change as a function

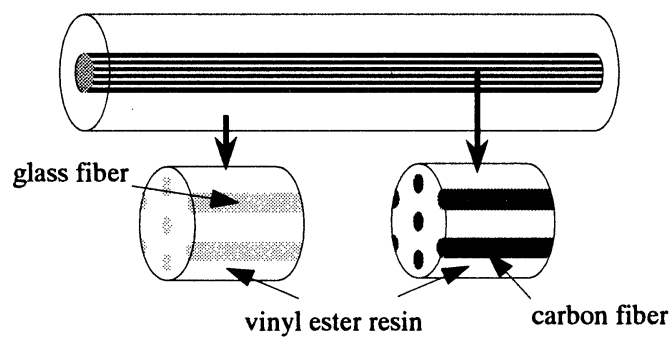

(a) carbon-fiber glass-fiber reinforced plastics of applied strain in tensile loading tests. The resistance change was defined as relative change in resistance $\left(R-R_{0}\right) / R_{0}$, indicated by $\Delta R / R_{0}$ in which $R_{0}$ denotes initial resistance before loading. The types of loading were in two ways; 1 ) a normal tensile test until specimen fracture, 2) cyclic loading-unloading test below the maximum stress level.

Figures 2 shows the changes in electrical resistance and applied stress for CFGFRP and CPGFRP as a function of applied strain in tensile test. The stresses in both specimens increased linearly in proportion to the strains until fracture of carbon fiber or glass fiber. The CFGFRP indicated slight change in resistance below $0.5 \%$ strain and tremendous change around $0.7 \%$ strain; namely, the resistance of CFGFRP exhibited non-linear response to applied strain as shown in Fig. 2 (a). The initial resistance $R_{0}$ for CPGFRP was higher than that for CFGFRP because of slight electrical contact between carbon particles in percolation structure. As

Fig. 1 Schematic drawings of the structural design for CFGFRP (a) and CPGFRP (b).
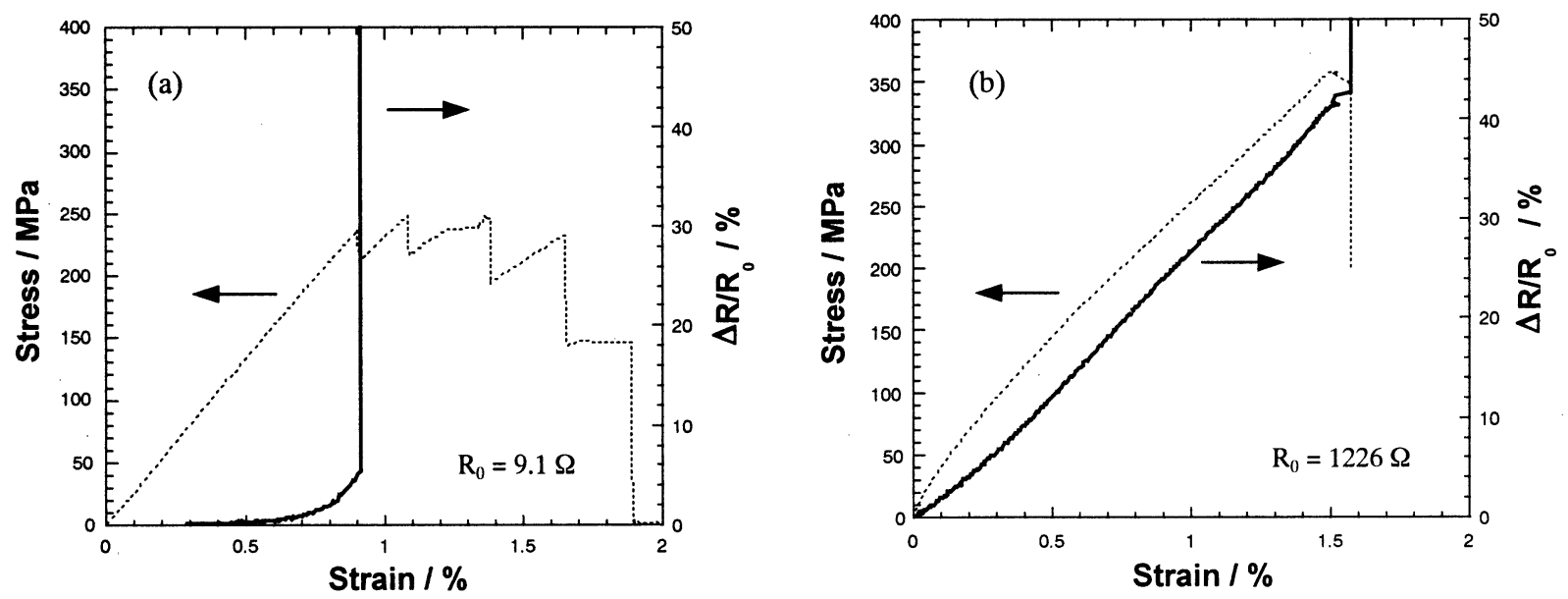

Fig. 2 Changes in electrical resistance (solid line) and applied stress (dashed line) as a function of applied strain in tensile tests for CFGFRP (a) and CPGFRP (b). 


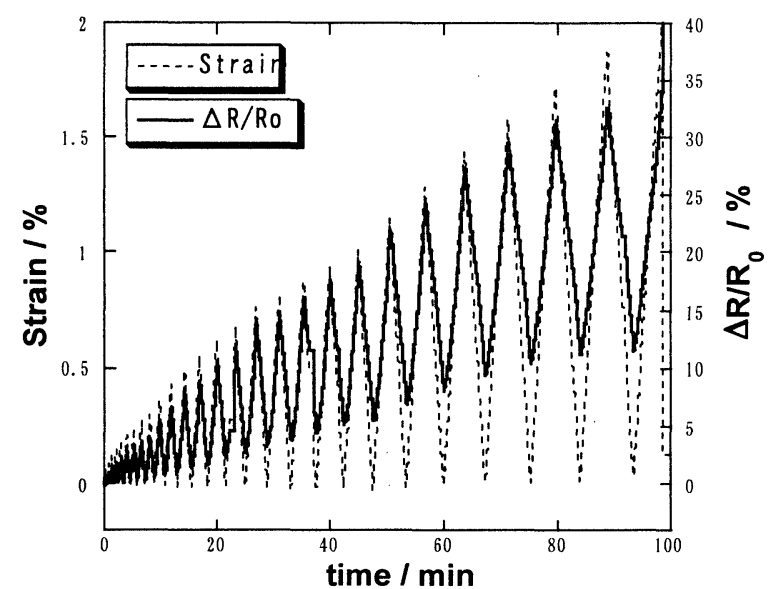

Fig. 3 Change in resistance (solid line) and applied strain (dashed line) as a function of time in cyclic loading tests for the CPGFRP.

can be seen from Fig. 2 (b), the CPGFRP indicated linear increase in resistance with increasing tensile strain. The response of the resistance to applied strain appeared around the strain of $0.01 \%$ (100 $\mu$ strain) or less. Comparing Fig. 2 (a) with (b) has shown that the CPGFRP possesses higher sensitivity at the small strain level and wider detectable strain range than CFGFRP. These results mean that the percolation structure formed with carbon particles enables more sensitive and adaptable diagnosis of damage than the structure consisting of carbon fiber. The fine response of resistance for CPGFRP were attributed to local break in electrical contact between carbon particles because of micro crack formation in the matrix or of rearrangement of the percolation structure under tensile stress.

Figure 3 shows resistance change and applied strain as a function of time in cyclic loading test for CPGFRP. ${ }^{7}$ Specimens were loaded and unloaded cyclically under a progressive increase in stress. It can be seen that the resistance change corresponded well with variation in strain. It is worthy of notice that a part of resistance change in the elongated composites remained after unloading despite elastic deformation. The residual resistance appeared after the application of $0.2 \%$ strain, and then increased with increase in maximum applied strain. The maximum resistance change during loading indicated by $\Delta R_{\max }$ and the residual resistance

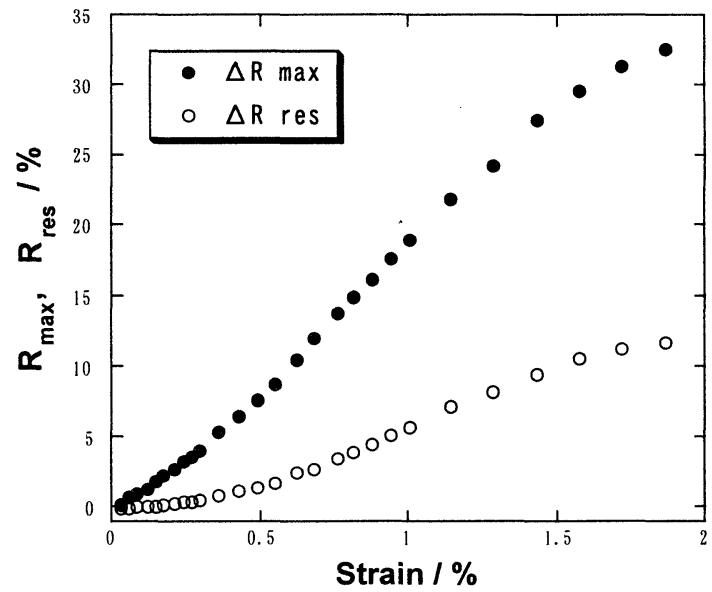

Fig. 4 Maximum resistance change at loading state and residual resistance change at unloading state as a function of applied strain in cyclic loading tests for the CPGFRP.

change after unloading denoted by $\Delta R_{\text {res }}$ were arranged with respect to maximum strain applied in the past as shown in Fig. $4 .^{7}$ The change in residual resistance correlated closely with previous maximum strain, suggesting that the CPGFRP has the ability to memorize maximum applied through the measurement of residual resistance. It seems likely that this phenomenon is due in part to the irreversible change in percolation structure. Although the elongation of CPGFRP removed elastically after unloading, the percolation structure must have not returned reversibly to initial state because of the crack formation in the matrix or the rearrangement of percolation structure.

\section{APPLICATION OF SELF-DIAGNOSIS FRP}

The FRP containing carbon particles was embedded in tensile side of mortar blocks in order to demonstrate the self-diagnosis function. Figure 5 shows applied load and resistance change of CPGFRP as a function of displacement in bending test. ${ }^{5}$ The embedded CPGFRP was located in $8 \mathrm{~mm}$ apart from tensile surface of mortar block of $4040160 \mathrm{~mm}$. The load-displacement curve indicated discontinuous change at the points of $A$ and $\mathrm{B}$, which corresponded to crack formation and propagation in the mortar block respectively. The crack formation and propagation were shown in photographs of mortar block. The resistance of 


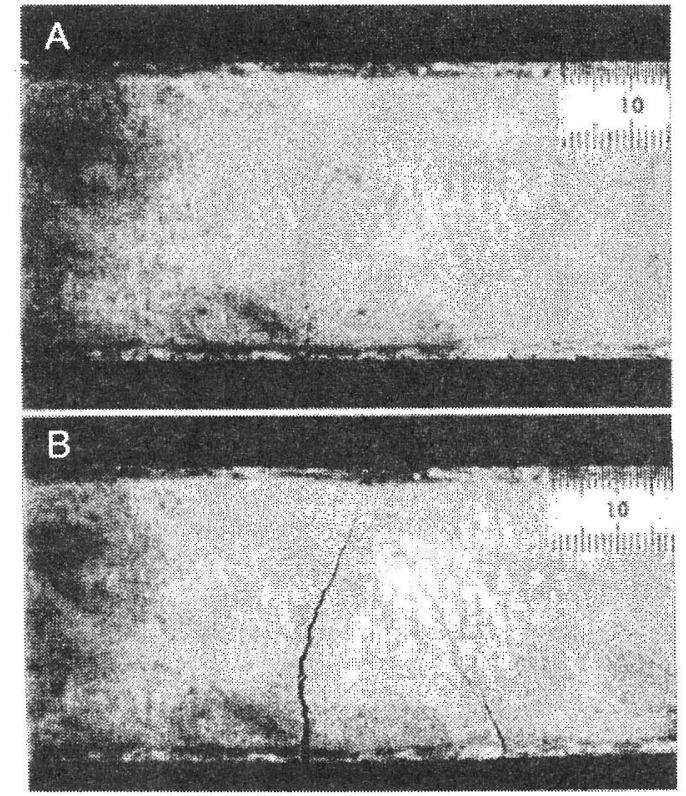

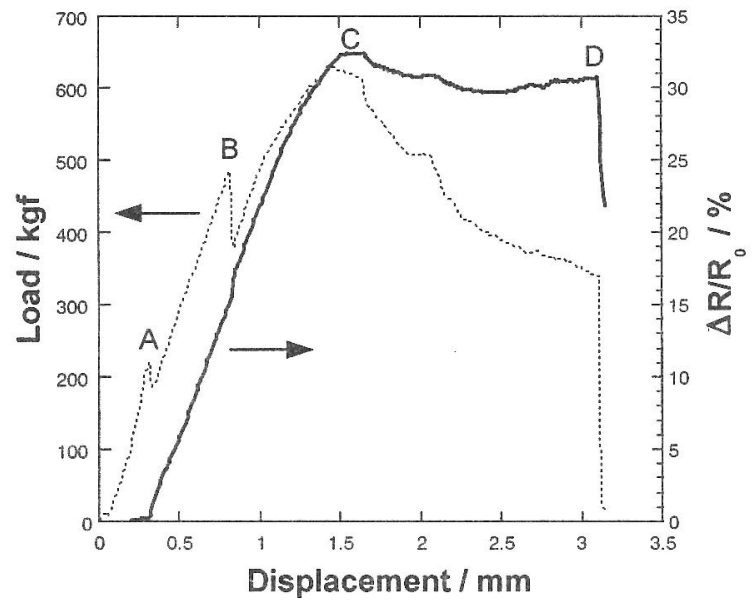

Fig. 5 Changes in resistance (solid line) and applied load (dashed line) in a bending test for CPGFRP embedded in mortar block. These points of A and $\mathrm{B}$ on the graph correspond with the left marked photographs of mortar block.
CPGFRP began to increase slightly before crack formation. Noteworthy is that the obvious increase in resistance appeared simultaneously with micro crack formation and discontinuous resistance change generated in response to crack propagation. The residual resistance was observed in the FRP material after unloading at the point of $\mathrm{D}$. The resistance change of embedded CPGFRP corresponded well with propagation of damage inflicted on mortar block. These results demonstrated that the embedded CPGFRP had the ability to diagnose micro crack formation/propagation and loading history in cement based structural materials.

\section{IV.SELF-DIAGNOSIS FUNCTION OF CMC}

Figure 6 is the schematic drawings of structural design for $\mathrm{CMC}$ materials. The composites were fabricated by the filament winding method using

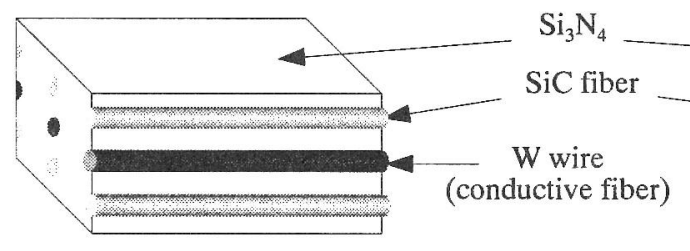

(a) $\mathrm{Si}_{3} \mathrm{~N}_{4}-\mathrm{SiC}$ fiber-W fiber

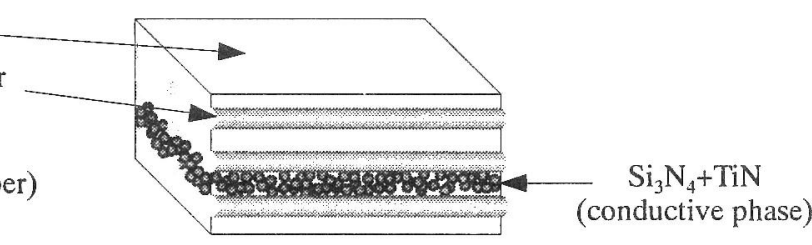

(b) $\mathrm{Si}_{3} \mathrm{~N}_{4}-\mathrm{SiC}$ fiber-( $\left.\mathrm{Si}_{3} \mathrm{~N}_{4}+\mathrm{TiN}\right)$

Fig. 6 Schematic drawings of the structural design for CMC containing W wire (a) and TiN particles (b).
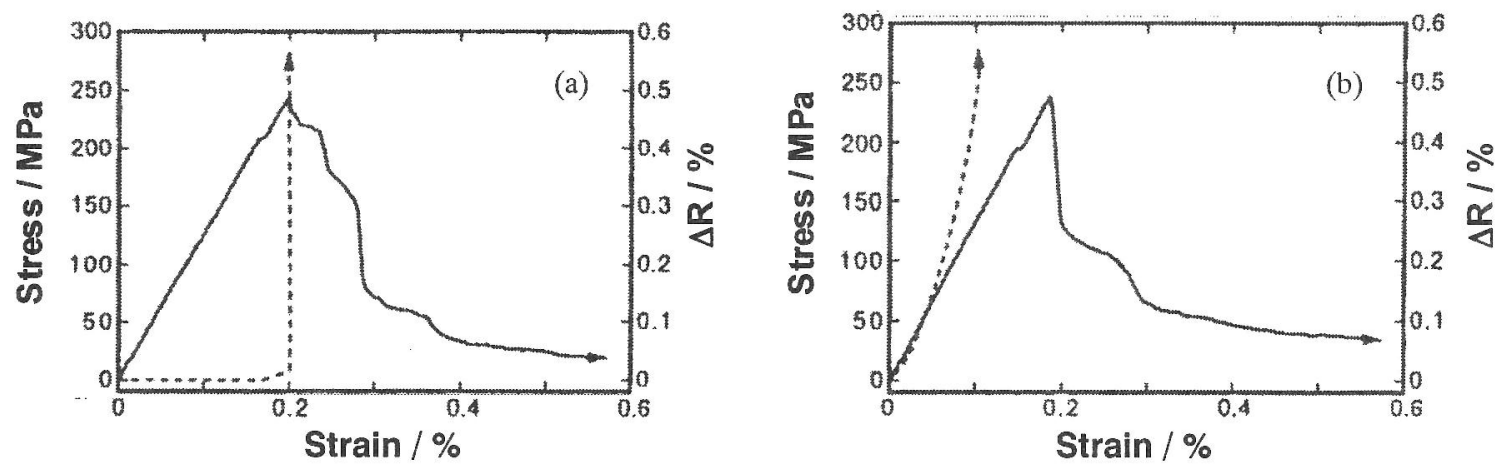

Fig. 7 Changes in electrical resistance (dashed line) and applied stress (solid line) as a function of applied strain in bending test for $\mathrm{CMC}$ with $\mathrm{W}$ wire (a) and with TiN particles (b). 
$\mathrm{Si}_{3} \mathrm{~N}_{4}$ particles (Ube Industries Co., Ltd. SN-COA) as the matrix and $\mathrm{SiC}$ fiber (Nippon Carbon Co., Ltd. NL-401) as the reinforcement for strengthening or toughening. A portion of the fibers was replaced with W wire (Nippon tungsten Co., Ltd. $\phi 30 \mu \mathrm{m}$ ). The conductive particles of $\mathrm{Si}_{3} \mathrm{~N}_{4}-40 \%$ TiN (Japan New Metals Co., Ltd.) were dispersed in a part of the matrix. These composites were hot pressed under $40 \mathrm{MPa}$ at $1773 \mathrm{~K}$ in $\mathrm{N}_{2}$ atmosphere for one hour. The sintered specimens were cut into bars of $3 \times 4 \times 45$ $\mathrm{mm}$ for bending test pieces, with either conductive wire or particles located $0.5 \mathrm{~mm}$ from tensile side.

The self-diagnosis functions of the CMC were evaluated by simultaneous measurements of stress and electrical resistance change $\Delta R$ as a function of applied strain in 4-pointed bending tests. The types of loading were in two ways; 1) a normal bending test until specimen fracture, 2) cyclic loadingunloading test below the maximum stress level.

The dependence of applied stress and change in resistance on strain for CMC are shown in Fig. $7 .^{3}$ Both composites indicated non-linear response of resistance change to applied strain and fractured at about $0.2 \%$ strain. The CMC with $\mathrm{W}$ wire showed slight change in resistance in a small strain level, and then drastic change were accompanied by own fracture as shown in Fig. 7 (a). The CMC containing TiN particles exhibited distinct change in resistance

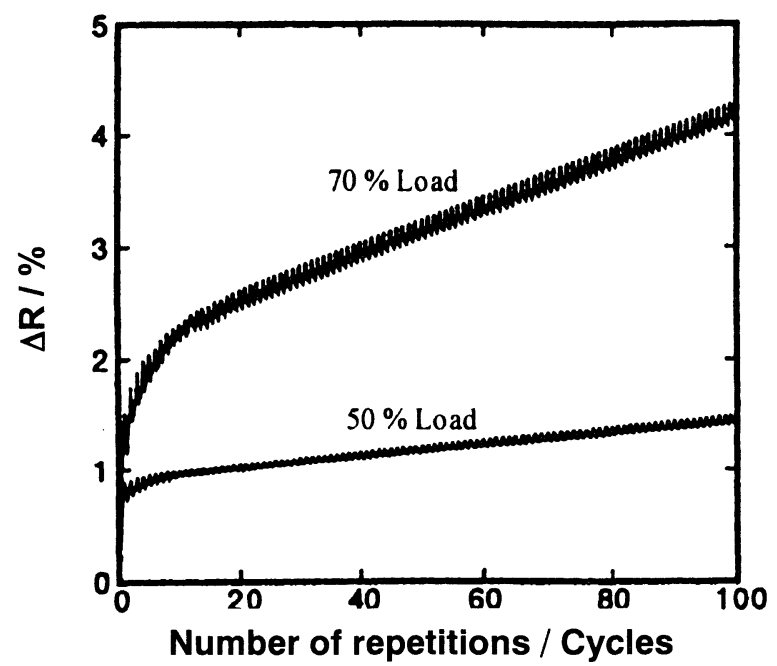

Fig. 8 Change in resistance of $\mathrm{CMC}$ containing TiN particles in cyclic bending test. from a small strain level to fracture in the composite as shown in Fig. 7 (b). These results suggest that the monitoring of the resistance for CMC with percolation structure is also advantageous to diagnose damages to the composites.

Figure 8 presents the variation of resistance for the $\mathrm{CMC}$ with TiN particles in cyclic bending test as a function of number of repetitions. ${ }^{3}$ The stress applied at loading state was kept constant at $50 \%$ and $70 \%$ of maximum stress (250 MPa) for the CMC. The residual resistance after unloading rapidly increased up to 10 cycles. It should be noted that the residual resistance proportionally increased with increasing number of repetitions after 20 cycles, which was distinct for cyclic loading under $70 \%$ of maximum stress. This result suggests that the CMC containing TiN particles have the ability to diagnose a cumulative fatigue for the composite by estimation of the residual resistance.

\section{v. CONCLUSION}

The self-diagnosis functions for several types of conductive composites have been investigated. Compared with the composites including conductive fiber or wire, the composites containing percolation structure were found to possess the advantages to diagnose deformation or damage in the composites by themselves. The FRP containing carbon particles in particular diagnosed micro-crack formation and propagation in cement based materials, showing that the self-diagnosis function of the FRP has the ability to monitor the health condition of concrete structures. Moreover, the FRP composites indicated the ability to memorize maximum applied through the measurement of residual resistance. The CMC materials also showed the ability to diagnose a cumulative fatigue for the composite by estimation of the residual resistance. It should be noted that these self-diagnosis functions are able to be easily obtained by simple measurement of electrical resistance.

It is expected that the percolation structure has the ability to be applied in other composite systems and the range of possible applications for self-diagnosis can be extended. 


\section{REFERENCES}

1 P-W. Chen and D. D. L. Chung, Compos. Part B, 27B [1], 11-23 (1996)

2 N. Muto, H. Yanagida, T. Nakatsuji, M. Sugita and Y. Ohtsuka, J. Am. Ceram. Soc., 76 [4], 87579 (1993)

3 H. Matsubara, M. Takada and H. Yanagida, Chemistry and Chemical Industry, 49 [1], 40-42 (1996)

4 Y. Arai, S.-G. Shin, M. Takada, M. Tsujii, H. Matsubara and H. Yanagida, Ceram. Mater. Syst. Compos. Struct., 99, 511-516 (1998)

5 M. Takada, S-G. Shin, H. Matsubara and H. Yanagida, J. Jpn. Soc. Compos. Mater., 25, 225230 (1999)
6 H. Yanagida and H. Matsubara, J. Jpn. Soc. Mech. Eng., 102 [963], 68-71 (1999)

7 S-G. Shin, H. Matsubara, Y. Okuhara, H. Yanagida and N. Takeda, Proc. 6th Jpn. Inter. SAMPE Symp., 2, 995-998 (1999)

8 Y. Okuhara, S.-G. Shin, H. Matsubara and H. Yanagida, Trans. Mater. Res. Soc. Jpn., 25 [2]581-584 (2000)

9 H. Nishimura, T. Sugiyama, Y. Okuhara, S.-G. Shin, H. Matsubara and H. Yanagida, Smart Structures and Materials 2000, 3985, 335 (2000)

Presented at 3rd Japan France Seminar on IMS 